\title{
Determinación de las emisiones de contaminantes del aire generadas por fuentes móviles en carreteras de Costa Rica
}

Jorge Herrera Murillo'

Susana Rodríguez Román²

José Félix Rojas Marín ${ }^{3}$

Fecha de recepción: 17 de enero del 2011

Fecha de aprobación: 16 de agosto del 2011

Herrera, J; Rodríguez, S; Rojas, J. Determinación de las emisiones de contaminantes del aire generadas por fuentes móviles en carreteras de Costa Rica. Tecnología en Marcha. Vol. 25, No I. Enero-Marzo 2012 Pág 54-63

I Laboratorio de Análisis Ambiental, Escuela de Ciencias Ambientales, Universidad Nacional. Campus Omar Dengo. Apartado 863000 Heredia, Costa Rica. Tel.: (506) 2277-3292. Correo electrónico: jherrer@una.ac.cr.

2 Escuela de Química, Universidad de Costa Rica. Ciudad Universitaria Rodrigo Facio, San Pedro de Montes de Oca, Costa Rica. Tel.: (506) 2207-5376. Correo electrónico: susanar@cariari.ucr.ac.cr

3 Laboratorio de Análisis Ambiental. Escuela de Ciencias Ambientales. Universidad Nacional. Campus Omar Dengo. Apto.: 86-3000 Heredia, Costa Rica. Tel.: (506) 2277-3292. Correo electrónico: jrojas60@yahoo.com 


\section{Palabras clave}

Fuentes móviles, emisiones, contaminantes criterio, Costa Rica.

\section{Resumen}

Se llevó a cabo una estimación de las emisiones generadas por la flota vehicular existente en Costa Rica a enero del 2010,mediante los factores de emisión obtenidos del modelo MOBILE 6 de la Agencia de Protección Ambiental de Estados Unidos y los datos de actividad reportados por la Dirección Sectorial de Energía. Del total de 544767 toneladas de contaminantes criterio producidas por la operación de fuentes móviles en carreteras, el 60,4\% corresponde a monóxido de carbono (CO), y son los vehículos particulares y de carga liviana que operan con gasolina las unidades más contaminantes, pues aportan un total del 73\% de las emisiones totales.

\section{Key words}

Mobile sources, emisions, criteria pollutants, Costa Rica.

\begin{abstract}
Emissions from vehicle fleet of Costa Rica at January 2010 were estimated using emission factors derivated from MOBILE 6 model (United States Environmental Protection Agency) and the activity data reported by the Energy Sector Management. The vehicle fleet in Costa Rica produced 544767 tons of criteria pollutants. Of these emissions, $60.4 \%$ correspond to carbon monoxide ( $\mathrm{CO}$ ) being passenger cars and light-duty gasoline-powered, the more polluting units, providing a $73 \%$ of total global emissions.
\end{abstract}

\section{Introducción}

Las emisiones de vehículos automotores están integradas por un gran número de contaminantes que provienen de muchos procesos diferentes (Figura I); de estas, se consideran con mayor frecuencia las emisiones del escape, resultantes de la combustión del combustible. Los contaminantes de interés clave en este tipo de emisiones incluyen: gases orgánicos totales (GOT), monóxido de carbono (CO), óxidos de nitrógeno (NOx), óxidos de azufre (SOx), material particulado (PM), gases tóxicos del aire (por ejemplo, I, 3-butadieno, benceno, formaldehído, etc.) y especies reductoras de visibilidad (e. g., amoniaco, sulfatos, PM2.5, etc.).

Además de las emisiones del escape, los vehículos automotores registran una gran variedad de procesos de emisión evaporativa que se limitan a emisiones de GOT e incluyen:

- Emisiones evaporativas del motor caliente: los cuales se presentan debido a la volatilización del combustible en el sistema de alimentación después de que el motor se ha apagado. El calor residual del motor volatiliza el combustible.
- Emisiones evaporativas de operación: Son las emisiones ocasionadas por las fugas de combustible, como líquido o vapor, que se presentan mientras el motor está en funcionamiento.

- Emisiones evaporativas durante la recarga de combustible: que constituyen las emisiones evaporativas desplazadas del tanque de combustible del vehículo durante la recarga. Estas pueden ocurrir mientras el vehículo está en reposo y en puntos conocidos, como las gasolineras. La recarga de combustible se maneja típicamente como fuente de área para efectos de los inventarios de emisiones.

- Emisiones diurnas: estas emisiones del tanque de combustible del vehículo son causadas por una mayor temperatura del combustible y la presión de vapor de este. Estas emisiones se deben al incremento de la temperatura ambiente ocasionado por el sistema de escape del vehículo o por el calor reflejado en la superficie del camino.

- Emisiones evaporativas en reposo: estas emisiones evaporativas son diferentes a las anteriores, 


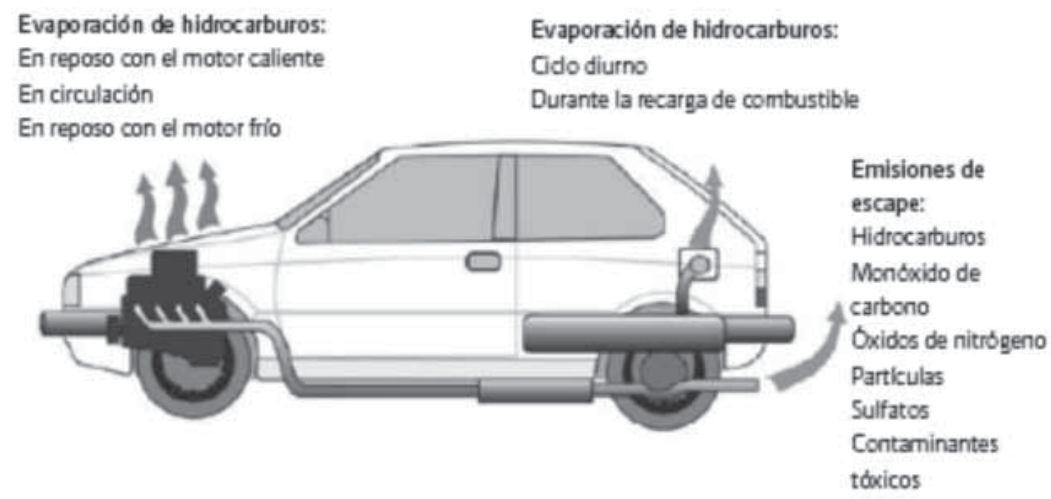

Fuente: INE-SEMARNAT, 2005.

Figura I. Emisiones generadas por vehículos automotores.

ya que se presentan cuando el motor no está en funcionamiento. Las pérdidas en reposo se deben principalmente a fugas de combustible y a la permeación del vapor a través de las líneas de alimentación del combustible (Radian, 1997).

Existen fuentes adicionales de emisiones de partículas (PM) de los vehículos automotores. Entre estas, la de mayor magnitud es el levantamiento de polvo del camino, que es el polvo recogido por las llantas del vehículo y suspendido en el aire por la turbulencia ocasionada por el movimiento. Las emisiones de polvo son manejadas, generalmente, como fuente de área en los inventarios de emisiones. Otras fuentes de PM no originadas en el escape incluyen el desgaste de las llantas y frenos. En general, estas fuentes son insignificantes cuando se comparan con las del escape y el polvo levantado y, por lo tanto, en algunas ocasiones son omitidas de los inventarios de emisiones (USEPA, 1992).

La ecuación básica utilizada para estimar las emisiones de los vehículos automotores involucra los datos de actividad vehicular y un factor de emisión.

$$
\mathrm{Ep}=\mathrm{KRV} \times \mathrm{FEp}
$$

Donde:

$E p=$ Emisiones totales del contaminante $p$

$\mathrm{KRV}=$ Kilómetros recorridos por el vehículo

FEp $=$ Factor de emisión del contaminante $p$

Para los vehículos automotores, los datos de actividad se refieren a los kilómetros recorridos por el vehículo (KRV), mientras que los factores de emisión se expresan en unidades de gramos de conta- minante por KRV. Los KRV representan la distancia total recorrida por una población de vehículos en un periodo de tiempo determinado. Es preferible que los KRV sean estimados a partir de modelos de transporte o de conteos de vehículos en circulación. En algunos casos, sin embargo, los KRV deben ser calculados a partir de las estadísticas de consumo de combustible. La ecuación básica de estimación presentada anteriormente es aplicable para la mayoría de los contaminantes gaseosos y partículas. Para otros contaminantes, tales como SOx y el plomo, las emisiones se calculan mediante un balance de combustible, con la suposición de que se emite la totalidad del azufre o plomo contenido en el combustible (Wark y Warne, 2007).

Los factores de emisión para los distintos contaminantes se derivan del uso de modelos. La razón de esto es que las emisiones de los vehículos automotores son más complejas y dinámicas que la mayoría de los otros tipos de fuentes de emisiones. Por ejemplo, los cambios en las características del combustible, las velocidades de operación del vehículo, la tecnología para el control de emisiones, la temperatura ambiente y la altitud pueden afectar los factores de emisión. Con el objeto de incorporar estos y otros factores, generalmente se utiliza un modelo de factor de emisión que incluye los efectos de numerosos parámetros.

El modelo MOBILE es un programa de cómputo integrado por rutinas elaboradas en lenguaje de programación Fortran y es utilizado para el cálculo de factores de emisión para distintos tipos de vehículos que operan tanto con motores de gasolina como de diesel. MOBILE calcula factores de emisión 
para veintiocho diferentes categorías vehiculares, las cuales son conformadas con base en criterios tales como el uso del vehículo, el tipo de combustible empleado, el peso bruto vehicular y la tecnología del motor (Tabla I). Adicionalmente, para cada categoría vehicular, MOBILE es capaz de estimar factores de emisión para veinticinco años-modelo, dentro de los años calendario de 1952 a 2050 (Hernández, 2009).

MOBILE estima los factores de emisión para hidrocarburos, monóxido de carbono, óxidos de nitrógeno, dióxido de carbono, material particulado (proveniente de varios componentes, como el desgaste de llantas y frenos, además de lo emitido por el escape del motor) y tóxicos como el benceno, metilterbutiléter, butadieno, formaldehído, acetaldehído y acroleína.

En el presente artículo se exponen los resultados del proceso de cálculo de las emisiones generadas por la flota vehicular existente en el país a enero del 2010 y su evolución en los últimos siete años como mecanismo para identificar posibles áreas de intervención para reducir emisiones y mejorar la calidad del aire en el país.

\section{Metodología}

\section{Flota vehicular}

En el cálculo de las emisiones contaminantes por fuentes móviles, se distribuyó la flota vehicular en circulación del país a enero del 2010 en seis categorías (tabla 2). La base de datos utilizada para realizar dicha clasificación se obtuvo del Departamento de Marchamos del Instituto Nacional de Seguros. A partir de estos datos, se realizaron las siguientes consideraciones:

- El parque vehicular que circula en el país tiene un rango de antigüedad de más de 30 años, sin embargo, debido a las necesidades del Modelo

Tabla I. Categorías vehiculares empleadas por MOBILE 6

\begin{tabular}{|c|c|c|}
\hline Número & Tipo de vehículo & Descripción \\
\hline I & LDGV & Vehículos ligeros de gasolina (autos de pasajeros) \\
\hline 2 & LDGTI & Camiones ligeros de gasolina I (PBV* de 0 a 2,722 kg; PP** de 0 a I,70। kg) \\
\hline 3 & LDGT2 & Camiones ligeros de gasolina 2 (PBV de 0 a 2,722 kg; PP > I,70। a 2,608 kg) \\
\hline 4 & LDGT3 & Camiones ligeros de gasolina 3 (PBV > 2,722 a 3,856 kg; PPA**** de 0 a 2,608 kg) \\
\hline 5 & LDGT4 & Camiones ligeros de gasolina 4 (PBV > 2,722 a 3,856 kg; PPA de 2,609 kg y mayores) \\
\hline 6 & HDGV2b & Vehículos pesados de gasolina clase $2 \mathrm{~b}$ (PBV > 3,856 a 4,536 kg) \\
\hline 7 & HDGV3 & Vehículos pesados de gasolina clase 3 (PBV > 4,536 a 6,350 kg) \\
\hline 8 & HDGV4 & Vehículos pesados de gasolina clase 4 (PBV > 6,350 a 7,258kg) \\
\hline 9 & HDGV5 & Vehículos pesados de gasolina clase 5 (PBV > 7,258 a 8,845 kg) \\
\hline 10 & HDGV6 & Vehículos pesados de gasolina clase 6 (PBV > 8,845 a II,794kg) \\
\hline 11 & HDGV7 & Vehículos pesados de gasolina clase 7 (PBV > 11,794 a 14,969kg) \\
\hline 12 & HDGV8a & Vehículos pesados de gasolina clase 8a (PBV > 14,969 a 27,216 kg) \\
\hline 13 & HDGV8b & Vehículos pesados de gasolina clase 8b (PBV > 27,216 kg) \\
\hline 14 & LDDV & Vehículos ligeros diesel (autos de pasajeros) \\
\hline 15 & LDDTI2 & Camiones ligeros de diesel I y 2 (PBV de 0 a $2,722 \mathrm{~kg}$ \\
\hline 16 & HDDV2b & Vehículos pesados de diesel clase $2 \mathrm{~b}$ (PBV de 3,856 a 4,536 kg \\
\hline 17 & HDDV3 & Vehículos pesados de diesel clase 3 (PBV > 4,536 a 6,350 kg) \\
\hline 18 & HDDV4 & Vehículos pesados de diesel clase 4 (PBV > 6,350 a 7,258 kg) \\
\hline 19 & HDDV5 & Vehículos pesados de diesel clase 5 (PBV > 7,258 a 8,845 kg) \\
\hline 20 & HDDV6 & Vehículos pesados de diesel clase 6 (PBV > 8,845 a I।,794kg) \\
\hline 21 & HDDV7 & Vehículos pesados de diesel clase 7 (PBV > I।,794 a 14,969kg) \\
\hline 22 & HDDV8a & Vehículos pesados de diesel clase $8^{\mathrm{a}}$ (PBV > 14,969 a 27,216 kg) \\
\hline 23 & HDDV8b & Vehículos pesados de diesel clase 8b (PBV > 27,216 kg) \\
\hline 24 & MC & Motocicletas (de gasolina) \\
\hline 25 & HDGB & Autobuses de gasolina (escolar y transporte urbano e inter-urbano ) \\
\hline 26 & HDDBT & Autobuses de transporte urbano e inter-urbano de diesel \\
\hline 27 & HDDBs & Autobuses escolares de diesel \\
\hline 28 & LDDT34 & Camiones ligeros de diesel 3 y 4 (PBV > 2,722 a 3856 kg) \\
\hline
\end{tabular}


Tabla 2. Distribución de la flota vehicular por año del modelo en Costa Rica a enero del 2010

\begin{tabular}{|c|c|c|c|c|c|c|c|c|c|c|c|c|}
\hline \multirow{2}{*}{$\begin{array}{c}\text { Año } \\
\text { Modelo }\end{array}$} & \multicolumn{2}{|c|}{ Automóviles } & \multicolumn{2}{|c|}{ Carga liviana } & \multicolumn{2}{|c|}{ Carga pesada } & \multicolumn{2}{|c|}{ Taxis } & \multicolumn{2}{|c|}{ Motocicletas } & \multicolumn{2}{|c|}{ Autobuses } \\
\hline & Gasolina & Diesel & Gasolina & Diesel & Gasolina & Diesel & Gasolina & Diesel & Gasolina & Diesel & Gasolina & Diesel \\
\hline anteriores & 28862 & 10406 & 9579 & 9380 & 32 & 4782 & 5 & 14 & 2587 & 0 & I & 5 \\
\hline 1986 & 10565 & 977 & 5031 & 2239 & 5 & 827 & 0 & 0 & 335 & 0 & 0 & 7 \\
\hline 1987 & 21850 & 1394 & 7896 & 2311 & 2 & 1183 & I & I & 516 & 0 & 0 & 5 \\
\hline 1988 & 26758 & 914 & 4485 & 1675 & 7 & 1304 & I & 0 & 532 & 0 & 4 & 11 \\
\hline 1989 & 21176 & 871 & 4853 & 2008 & 9 & 1443 & I & I & 744 & 0 & 0 & 51 \\
\hline 1990 & 22220 & 1195 & 4770 & 2645 & 6 & 1478 & 0 & 2 & 1045 & 0 & 7 & 200 \\
\hline 1991 & 34882 & 1010 & 3852 & 1949 & 6 & 1111 & 2 & I & 1044 & 0 & 12 & 270 \\
\hline 1992 & 44291 & 1846 & 3185 & 1469 & 6 & 1234 & 2 & 4 & 1217 & I & 9 & 371 \\
\hline 1993 & 43123 & 2666 & 3413 & 2632 & 9 & 1777 & 24 & 0 & 1718 & 0 & 14 & 509 \\
\hline 1994 & 43069 & 2839 & 3468 & 2708 & 11 & 1747 & 1941 & 122 & 2574 & 0 & 12 & 821 \\
\hline 1995 & 36465 & 3169 & 3000 & 2784 & 13 & 2523 & 1509 & 102 & 1915 & 0 & 9 & 1044 \\
\hline 1996 & 29281 & 2431 & 1667 & 1721 & 6 & 2028 & 869 & 86 & 1823 & I & 12 & 886 \\
\hline 1997 & 26664 & 3696 & 1709 & 2018 & 8 & 1725 & 843 & 92 & 1462 & 0 & 7 & 865 \\
\hline 1998 & 21709 & 2217 & 2348 & 2737 & 25 & 2113 & 394 & 43 & 1940 & 0 & 11 & 746 \\
\hline 1999 & 22349 & 2719 & 1554 & 4091 & 28 & 2196 & 483 & 69 & 1106 & I & 6 & 982 \\
\hline 2000 & 22672 & 2549 & 1696 & 3549 & 23 & 2247 & 938 & 81 & 1243 & 0 & 4 & 1099 \\
\hline 2001 & 21103 & 3207 & 1080 & 3015 & 17 & 1074 & 783 & 60 & 1363 & 0 & 6 & $106 \mid$ \\
\hline 2002 & 18612 & 3529 & 910 & 3407 & 6 & 569 & 655 & 124 & 2150 & 0 & 4 & 975 \\
\hline 2003 & 15830 & 3102 & 973 & 4121 & 6 & 1025 & 233 & 63 & 2542 & I & 2 & 1083 \\
\hline 2004 & $|274|$ & 2852 & 585 & 3186 & 7 & 428 & 171 & 261 & 3220 & I & 5 & 720 \\
\hline 2005 & 12878 & 3832 & 597 & 3507 & 4 & 304 & 187 & 583 & 5660 & 4 & 2 & 926 \\
\hline 2006 & 13472 & 4935 & 860 & 5066 & 7 & 274 & 57 & 826 & 15262 & 85 & 2 & 762 \\
\hline 2007 & 15320 & 6715 & 636 & 6855 & 4 & 502 & 75 & 606 & 24512 & 7 & 0 & 1111 \\
\hline 2008 & 22894 & 8188 & 785 & 11238 & 24 & 988 & 69 & 627 & 41309 & 47 & 4 & 1138 \\
\hline 2009 & 15477 & 3111 & 495 & 6100 & 72 & 516 & 146 & 424 & 27888 & 38 & 11 & 666 \\
\hline 2010 & 3660 & 720 & 85 & 859 & 0 & 69 & 7 & 2 & 4898 & 0 & 0 & 244 \\
\hline Total & 607922 & 81090 & 69513 & 93271 & 342 & 35468 & 9398 & 4194 & 150603 & 187 & 143 & 16558 \\
\hline
\end{tabular}

MOBILE 6, fue necesario acotar la clasificación a veinticinco años de modelos.

- Las unidades de clasificación utilizadas corresponden a: automóviles, carga liviana, carga pesada, autobuses, taxis y motocicletas.

- Para los efectos del cálculo de factores de emisión, se consideraron los tractocamiones como parte de la categoría de carga pesada, al igual que los remolques, los cuales se contaron como unidades independientes.

- Se asume que los vehículos que circulan en el país tienen actividad los 365 días del año, con lo cual se ignora el programa de restricción vehicular existente en la ciudad de San José, debido a que su área de cobertura es mínima con respecto al área de estudio.

\section{Actividad vehicular:}

Para el presente estudio, los datos de actividad fueron tomados de la Encuesta del recorrido medio anual de los vehículos en circulación en Costa Rica, elaborada por la Dirección Sectorial de Energía de Costa Rica (DSE, 2009) (tabla 3). En este estudio se utilizaron cuatro métodos de cálculo para el recorrido anual en kilómetros:

I. Estimación según sticker y odómetro: consiste en hacer un cálculo del recorrido tomando en cuenta la fecha del último cambio de aceite al automotor y la marca del odómetro al próximo cambio de aceite. 
2. Estimación según la declaración de la marca del odómetro cuando se compró el automotor y el día de la entrevista: en este método de cálculo se consulta si el vehículo era nuevo o usado, y si el conductor sabía la marca del odómetro el día de la compra.

3. Estimación según la declaración del rendimiento del vehículo en kilómetros por galón: en este método se le pregunta al conductor por el rendimiento en kilómetros por galón en forma general, en la ciudad y en carretera, también se pregunta por el gasto en colones de combustible por semana que tiene el conductor con ese vehículo.

\section{Estimación según la llamada posterior al con-} ductor: consiste en solicitar al conductor del automotor la posibilidad de llamarle varios días después para que declare la marca del odómetro de ese día, entonces, con la diferencia entre la marca del odómetro el día de la entrevista y la marca del odómetro el día de la llamada, dividido por el número de días transcurridos entre ambas fechas, se calcula un recorrido diario y la estimación anual respectiva.

Para obtener un dato único de recorrido anual en kilómetros para cada automotor, se calculó un promedio entre los datos que se estimaron según los cuatro métodos.

\section{Factores de emisión}

Los factores de emisión para los contaminantes criterio, incluidos en el alcance de este inventario, fueron obtenidos a partir del software MOBILE 6, que es un modelo computacional desarrollado por la US EPA (Agencia de Protección Ambiental de Estados Unidos) y modificado de acuerdo con las condiciones presentes en Costa Rica. En la tabla 4 se muestra la información requerida por el modelo MOBILE 6 para el cálculo de los factores de emisión. Además, el modelo MOBILE 6 requiere información representativa del parque vehicular del área metropolitana, el cual está constituido por los siguientes datos (USEPA, 1992):

Tabla 3. Datos de la actividad de la flota vehicular que circula en Costa Rica

\begin{tabular}{|c|c|}
\hline Categoría de vehículo & $\begin{array}{c}\text { Kilómetros promedio } \\
\text { recorridos en un año }\end{array}$ \\
\hline Automóvil particular & 20768 \\
\hline Carga liviana & 27990 \\
\hline Carga pesada & 38934 \\
\hline Taxis & 86109 \\
\hline Buses & 43417 \\
\hline Motos & $1609 \mid$ \\
\hline
\end{tabular}

Fuente: Dirección Sectorial Energía, 2010

Tabla 4. Información requerida como datos de entrada para la operación del modelo MOBILE 6

\begin{tabular}{|c|c|}
\hline Parámetro & Datos de entrada proporcionados \\
\hline Región & $\begin{array}{c}\text { Ciudad a una altitud menor } \\
\text { o igual a } 5500 \mathrm{ft} \mathrm{(I677} \mathrm{m)}\end{array}$ \\
\hline Año calendario por evaluar & 2010 \\
\hline Velocidad promedio de circulación en la región & $33, \mathrm{l} \mathrm{km} / \mathrm{h} \mathrm{(I)}$ \\
\hline Temperatura máxima diaria para la región & $25,8^{\circ} \mathrm{C} \mathrm{(2)}$ \\
\hline Temperatura mínima diaria para la región & $17,4{ }^{\circ} \mathrm{C}(2)$ \\
\hline Presión de vapor reid de la gasolina & $61,5 \mathrm{kPa}(3)$ \\
\hline Contenido de azufre en diesel & $400 \mathrm{ppm}(3)$ \\
\hline Contenido de azufre en gasolina & $300 \mathrm{ppm}(3)$ \\
\hline Contenido de oxigenados en gasolina & $0,42 \%(3)$ \\
\hline
\end{tabular}

I. Dato tomado del Estudio de Oferta y demanda de Transporte, PRUGAM (2007).

2. Promedio de las estaciones del Instituto Meteorológico Nacional ubicadas en la zona.

3. Información proporcionada por la Refinadora Costarricense de Petróleo. 
- Fracciones de participación de la flota por el tipo de vehículo y año del modelo.

- Fracciones de los kilómetros recorridos por el tipo de vehículo y año del modelo.

Mediante las aplicaciones del modelo se obtuvieron los factores de emisión para cada contaminante, por tipo de vehículo, combustible y año del modelo. En forma adicional, se realizó una homologación de las categorías de clasificación de la flota vehicular que circula en Costa Rica con respecto a las propuestas en el modelo MOBILE 6, de acuerdo con las directrices indicadas en la tabla 5.

\section{Resultados y discusión}

\section{Factores de emisión}

Tal como se puede observar en la figura 2, los factores de emisión para GOT en los vehículos que operan con gasolina (autos particulares y motocicletas) son mucho mayores que los obtenidos para vehículos de diesel (autobuses y carga pesada). A diferencia de los motores de gasolina, los motores diesel, en general, presentan emisiones más bajas de GOR y, por tanto, menores emisiones de GOT. Esto se debe a dos razones básicas: en primer lugar, los motores diesel operan con relaciones elevadas aire/combustible, lo que permite que existan concentraciones de oxígeno en exceso y altas temperaturas durante e inmediatamente después del proceso de combustión, lo que favorece la combustión completa de los

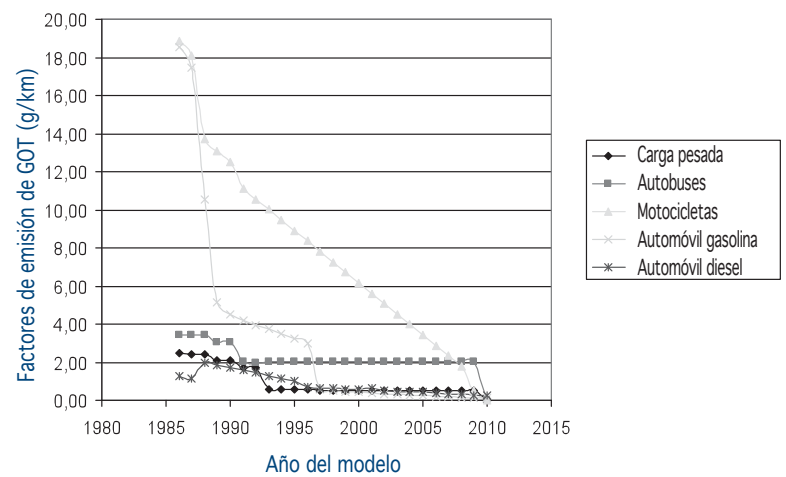

Figura 2. Factores de la emisión de GOT, generada por fuentes móviles, obtenidos con el modelo MOBILE 6 para la flota vehicular de Costa Rica.

GOR. En segundo lugar, el diesel es un combustible relativamente no volátil, lo que hace que las emisiones de evaporación por el uso de combustible diesel sean significativamente menores con relación a la gasolina.

De igual forma, si se analiza el comportamiento de los factores de emisión para óxidos de nitrógeno (figura 3), se puede observar que se registran valores superiores para vehículos que operan con diesel. Estas unidades no cuentan con un convertidor catalítico para NOx, lo que provoca que las emisiones de NOx sean mayores. Por otro lado, el diesel utiliza más aire en su combustión, por lo que tiene mayores cantidades de nitrógeno y, por lo tanto, causa mayores emisiones. Por último, el diesel tiene mayor poder calorífico, lo que hace que aumenten las

Tabla 5. Emisiones generadas por las fuentes móviles en Costa Rica, 2010

\begin{tabular}{|c|c|c|c|c|c|c|c|c|}
\hline \multirow{2}{*}{ Tipo de vehículo } & \multicolumn{8}{|c|}{ Emisiones (ton/año) } \\
\hline & PMIO & PM2,5 & GOT & GOR & $\mathrm{SO} 2$ & NOx & $\mathrm{CO}$ & $\mathrm{NH3}$ \\
\hline $\begin{array}{c}\text { Automóviles } \\
\text { gasolina }\end{array}$ & 217,2 & 86,9 & 52212 & 36026,3 & 70,8 & 30371 & 137258 & 794,7 \\
\hline Automóviles diesel & $|66|$, & 124,6 & 1270 & 478,8 & 168,4 & 2313 & 909 & 7,3 \\
\hline Taxis gasolina & $|4|$, & 5,64 & 1443 & 575,8 & 4,6 & 1996 & 7047 & 51,7 \\
\hline Taxis diesel & 34,9 & 26,2 & 156 & 62,4 & 35,6 & 246 & 157 & 1,5 \\
\hline Motocicletas & 56,4 & 22,6 & 7688 & 3189 & 63,0 & 2317 & 36106 & 16,8 \\
\hline Autobuses & 173,2 & 129,9 & 1439 & 777,1 & 257,1 & 16874 & $5|4|$ & $|2|$, \\
\hline Carga liviana diesel & 612,3 & 459,2 & 3731 & 1007,4 & 627,8 & 17536 & 6570 & 42,7 \\
\hline $\begin{array}{c}\text { Carga liviana } \\
\text { gasolina }\end{array}$ & 35,9 & 14,4 & 6005 & 4023 & 132,3 & 4448 & 125835 & 119,8 \\
\hline Carga pesada & 333,8 & 250,3 & 1632 & 783,4 & 290,2 & | | 442 & 10219 & 23,3 \\
\hline TOTAL & 1643,9 & 1119,7 & 75576 & 46923 & 1649,8 & 87543 & 329242 & 1069,9 \\
\hline
\end{tabular}


emisiones de NOx dado que es un contaminante susceptible a las altas temperaturas.

Las motocicletas y los vehículos livianos que operan con gasolina son las unidades de la flota vehicular que presentan los mayores factores de emisión de monóxido de carbono (figura 4) al ser motores menos eficientes, en términos de combustión, que aquellos operados con diesel.

\section{Emisiones generadas por las fuentes móviles}

En la tabla 5 se muestran las emisiones causadas por las fuentes móviles en las carreteras de Costa Rica, por contaminante y tipo de vehículo, en el 20 I O. En la figura 5 se puede observar cómo la mayor cantidad de las emisiones de partículas por fuentes móviles es aportada por los vehículos de carga liviana, los cuales

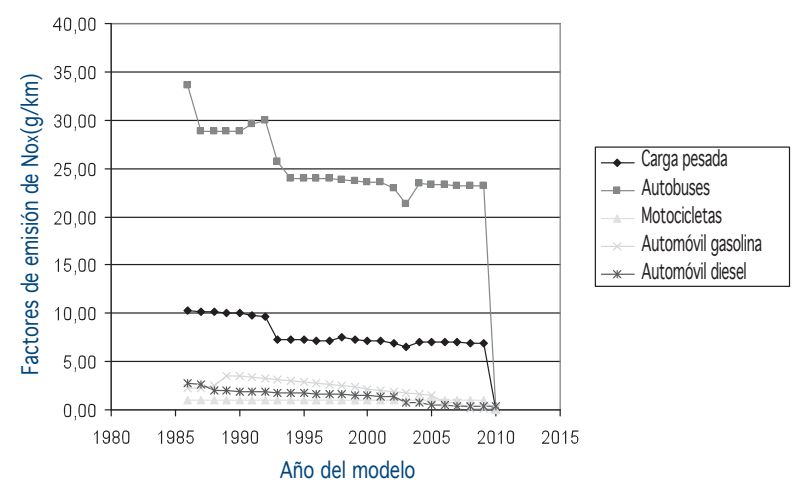

Figura 3. Factores de la emisión de NOx, generada por fuentes móviles, obtenidos con el modelo MOBILE 6 para la flota vehicular de Costa Rica.

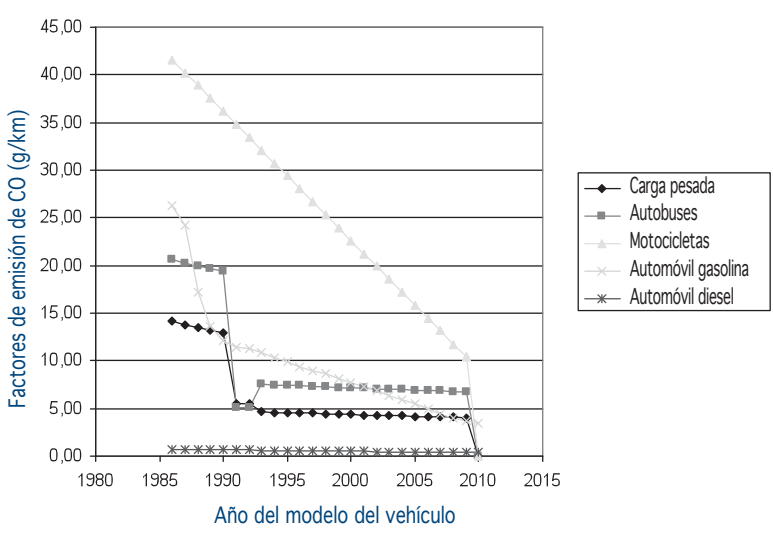

Figura 4. Factores de la emisión de $\mathrm{CO}$, generada por fuentes móviles, obtenidos con el modelo MOBILE 6 para la flota vehicular de Costa Rica. generan el mayor porcentaje de toneladas al año de partículas $\mathrm{PM}_{10}$ y $\mathrm{PM}_{2,5}$ (37\% y $41 \%$ respectivamente). Una situación diferente se presenta al analizar las emisiones de $\mathrm{NOx}$ y $\mathrm{CO}$, en donde los vehículos particulares resultaron ser la principal fuente emisora de estos gases resultantes del proceso de combustión, tal como se puede apreciar en la figura 6.

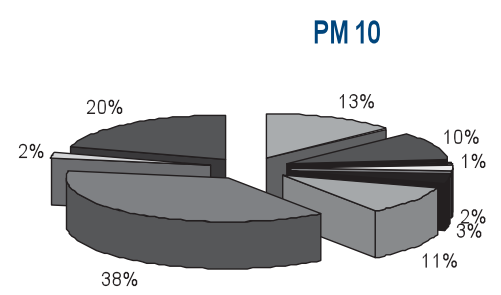

\begin{tabular}{|l|}
\hline Automóviles gasolina \\
$\square$ Automóviles diesel \\
$\square$ Taxis gasolina \\
$\square$ Taxis diesel \\
Motocicletas \\
$\square$ Autobuses \\
$\square$ Carga liviana diesel \\
$\square$ Carga liviana gasolina \\
Carga pesada
\end{tabular}
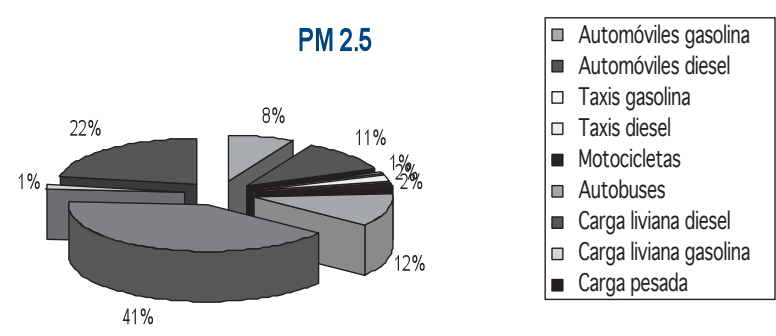

Figura 5. Distribución de las emisiones de material particulado generadas por las fuentes móviles en Costa Rica, según la categoría de vehículo.
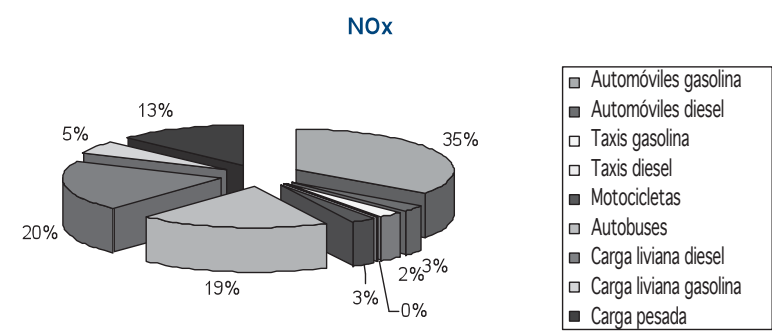

CO

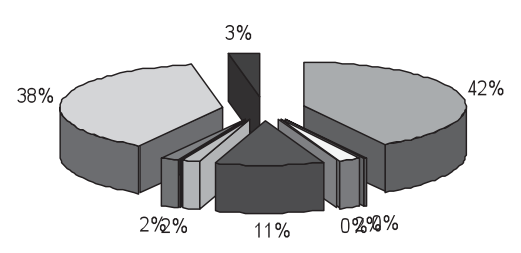

Figura 6. Distribución de las emisiones de NOx y CO generadas por las fuentes móviles en Costa Rica, según la categoría de vehículo. 
En la figura 7 se presenta la comparación porcentual entre la emisión de los precursores de ozono (gases orgánicos reactivos y óxidos de nitrógeno), así como el porcentaje de vehículos de diesel, que se encontraban en circulación en Costa Rica en el 2010, de acuerdo con el año del modelo. Se observa que el $45 \%$ de los vehículos (modelos 1993 y anteriores), emite el $62 \%$ de los gases orgánicos reactivos y el $42 \%$ de los óxidos de nitrógeno. Los vehículos cuyo modelo va de 1994 a 1997 (tecnología EPA 94) representan el $16 \%$ de la flota vehicular, los cuales emiten el 14\% de los gases orgánicos reactivos y el $22 \%$ de los óxidos de nitrógeno.

Finalmente, los vehículos que corresponden a los modelos 1998-2008 (tecnología EPA 98) representan el $39 \%$ de la flota de vehículos de diesel y aportan el $25 \%$ y el $35 \%$ de los gases orgánicos reactivos y de los óxidos de nitrógeno, respectivamente. Para complementar este análisis, en la figura 8 se consideró el resto de la flota, es decir, vehículos que utilizan gasolina como combustible, de acuerdo con el año del modelo correspondiente a los estratos tecnológicos y su aporte de emisiones de GOR y NOx.

En esta figura se observa que los vehículos que no cuentan con sistemas para controlar emisiones (modelos de 1990 y anteriores) representan el 20,7\% de los vehículos, y contribuyen con el $77,4 \%$ y el $22,7 \%$ de las emisiones de GOR y de NOx, respectivamente; el I I\% de los vehículos corresponde a los años 199| y 1992 que ya tienen incorporados algunos sistemas de control de emisiones, como encendido electrónico y convertidor catalítico de dos vías, los cuales emiten el 6,3\% de los gases orgánicos reactivos y el 18,2\% de óxidos de nitrógeno.

Por último, los vehículos correspondientes a los modelos de 1993 a 2008 (68\%), los cuales se consideran los menos contaminantes debido a que están equipados con sistemas de control de emisiones avanzados, tales como convertidor catalítico de tres vías, canister para controlar las emisiones evaporativas, computadoras a bordo y sensores de oxígeno, aportan el I8\% de los gases orgánicos reactivos y el 59\% de los óxidos de nitrógeno.

\section{Evolución de las emisiones (2003-2010)}

$\mathrm{Tal}$ como se puede observar en la figura 9, la tasa de crecimiento anual de las emisiones vehiculares de $\mathrm{CO}$, GOT y NOx corresponden a 9,6\%, I I, I\% y I4,3\% respectivamente, en el periodo 2003-20 I O. Si se analiza el comportamiento de las tasas, se puede observar una ligera disminución de las mismas en el periodo
2005-2010 en comparación con el periodo 20032005, lo cual puede ser razonablemente atribuido al efecto de la revisión técnica vehicular como mecanismo de control de las emisiones.

En el caso particular de las emisiones de $\mathrm{SO}_{2}$, los esfuerzos realizados por la Refinadora Costarricense de Petróleo por reducir el contenido de azufre en el diesel han permitido alcanzar una disminución sostenida en las emisiones de este contaminante, principalmente a partir del 2007 (figura 10).

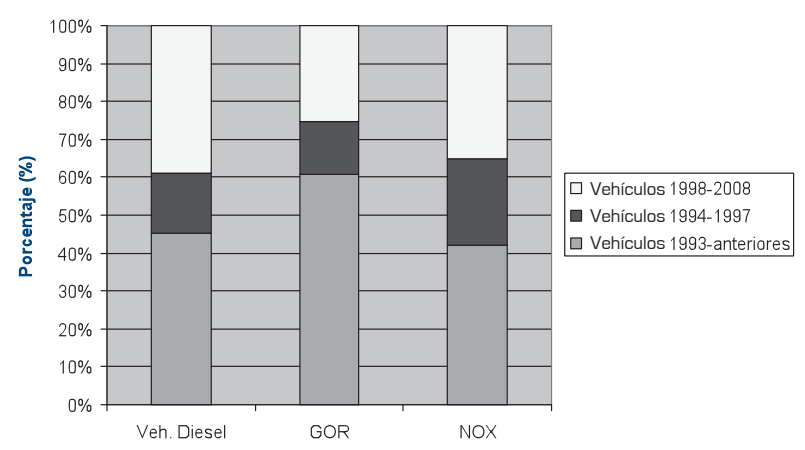

Figura 7. Contribución porcentual de las emisiones de GOR y NOx de los vehículos que operan con diesel en Costa Rica, 2010.

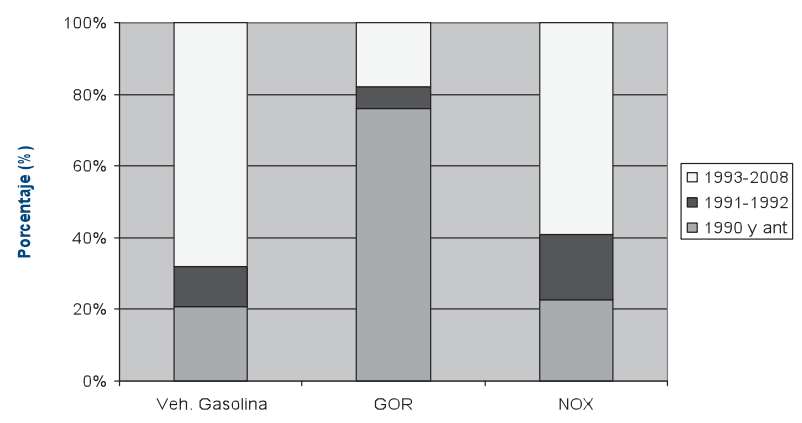

Figura 8. Contribución porcentual de las emisiones de GOR y NOx de los vehículos que operan con gasolina en Costa Rica, 2010.

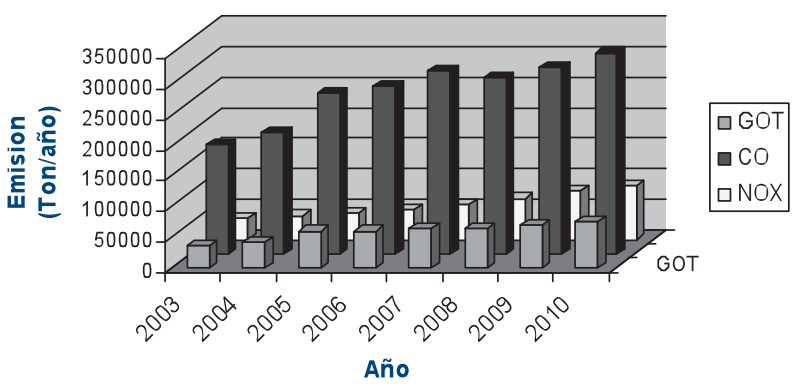

Figura 9. Evolución de las emisiones de GOT, $\mathrm{CO}$ y NOx generadas por fuentes móviles en Costa Rica (2003 - 20I0). 


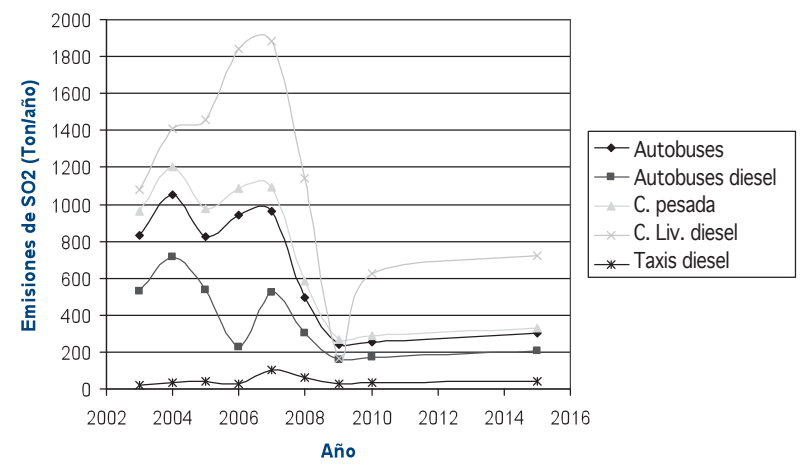

Figura 10. Evolución de las emisiones de dióxido de azufre por tipo de vehículo en el periodo 2003-2010.

\section{Conclusiones}

En Costa Rica se genera un total de 544767 toneladas de contaminantes criterio al año producidas por la operación de fuentes móviles en carreteras. El principal contaminante generado es el monóxido de carbono, el cual contribuye en un $60,4 \%$ a la totalidad de las emisiones. Las unidades más contaminantes de la flota son los vehículos particulares y de carga liviana que operan con gasolina, ya que aportan un total del $73 \%$ de las emisiones, al ser estas las más numerosas. Sin embargo, sobresalen en este análisis los autobuses y los vehículos de carga pesada, los cuales son menos del 5,8\% de la flota, pero emiten el $31 \%$ y $33 \%$ de las partículas $\mathrm{PM}_{10}$ y $\mathrm{SO}_{2}$, respectivamente.

\section{Bibliografía}

Dirección Sectorial de Energía. (2009). Encuesta del recorrido medio anual de los vehículos en circulación en Costa Rica 2009. Recuperado de http://www.dse. go.crles/03Publicaciones/04Tecnicas/RecorridoVehículosCR2009.pdf

Hernández, A. (2009). Evaluación del Plan Verde en Materia de Aire con base en el contenido de la Evaluación Ambiental Estratégica y modelación de los escenarios. Tesis de Maestria. Universidad Autónoma Metropolitana, Mexico D.F.

Radian. (1997, 2 I marzo). México Emissions Inventory Program Manuals, Volume V B Mobile Source Inventory Development, Final. Sacramento, California.

US EPA. (2002). User's Guide to MOBILE6.I and MOBILE6.2: Mobile Source Emission Factor Model (Guía del usuario del modelo de factores de emisión de fuentes móviles MOBILE6.I y MOBILE6.2). Recuperado de: http://www.epa.gov/otaq/models/mobile6/r02028.pdf.

Wark, K. \& Warner, C.F. (2007). Contaminación del Aire, orígen y control. México: Limusa. 